\title{
Improved Method of Ink-Gelatin Perfusion for Visualising Rat Retinal Microvessels
}

\author{
Tong Jianbin 1,*, Huang Liang, ${ }^{1, *}$, Huang Jufang ${ }^{1}$, Wang Hui ${ }^{1}$, Chen Dan ${ }^{1}$, \\ Zeng Leping ${ }^{1}$, Zhou Jin ${ }^{1}$ and Luo Xuegang ${ }^{1}$ \\ ${ }^{1}$ Department of Anatomy \& Neurobiology, Xiangya School of Medicine, Central South University, Changsha, 410013, China
}

Received April 28, 2008; accepted July 17, 2008; published online August 26, 2008

To visualize completely rat retinal microvessels, the gelatin-ink perfusion condition was systematically optimized using von Willebrand factor (vWf) immunostaining as control. Whether the vessel showed by the new perfusion condition can be used for double label with neurons or glial cells in the same retina was also tested. Our results showed that infusing rats first with $20 \mathrm{ml}$ of $37^{\circ} \mathrm{C}$ ink plus $3 \%$ gelatin at $140 \%$ rat mean arterial pressure (MAP), and subsequently with $20 \mathrm{ml}$ of $37^{\circ} \mathrm{C}$ ink plus $5 \%$ gelatin at $180 \%$ rat MAP allowed the ink to completely fill the rat retinal microvessels. Rat retinal microvessels labeled by the perfusion method were more in number than that by vWf immunostaining. Moreover, our data, for the first time, displayed that the improved gelatin-ink perfusion had no effect on and caused no contamination to the following fluorogold labeling or immunostaining of retinal neurons or glial cells in the same tissue. These data suggest that the improved gelatin-ink perfusion technique is a superior method for morphological characterization of rat retinal microvessels, compatible to the double labeling of glial cells and neurons, and it extends the practical scale of the classic method.

Key words: ink perfusion, von Willebrand factor, microvessel, retina, rat

\section{Introduction}

Retinopathies, such as diabetic retinopathy, are major causes of blindness and visual loss. The pathogenesis of some retinopathies with a local or systemic origin is closely associated with structural and functional alterations of the retinal microvasculature. Although the initiating factors and early stages of retinopathies vary, pathogenesis results in impairment of the blood supply, particularly oxygen transport and delivery [4]. The main function of retinal microvessels is to provide retinal tissue with oxygen and to respond to changing oxygen demands $[10,11,24]$. Therefore, identifying the relationships between retinal microvascular changes and the damage to retinal neurons and glial cells are very im-

Correspondence to: Huang Jufang and Luo Xuegang, Department of Anatomy \& Neurobiology, Xiangya School of Medicine, Central South University, Changsha, 410013, China.

E-mail: jufanghuang@yahoo.com.cn (Huang Jufang);

xgluo@xysm.net (Luo Xuegang)

*who made the same contribution to the article. portant for the understanding and therapeutic evaluation of retinopathies.

Currently, retinal microvessels are identified using a number of imaging techniques, each with specific disadvantages. These detection techniques include: histochemical assays that target enzymes enriched in blood vessels [3, 18], immunohistochemistry for vascular associated antigens (i.e. von Willebrand factor: a substance synthesised by, stored in, and released by endothelial cells) $[2,9,20]$, perfusion with ink $[1,5]$, and use of fluorescein sodium [14, 21, 28]. Histochemical assays and immunohistochemistry are limited to investigations of retinal microvessel changes and neurons or glial cells damage in the same archival rat retina. This limitation is partly due to the fact that thickness of nerve fibre layer is thinner in the periphery than that in the central which affects the results of immunostaining and the detection of enzymes' activity. Fluorescein sodium labeling fades easily and is not suitable for use in animals that lack retinal pigments [16]. These disadvantages limit the use of these labeling methods.

Simple ink perfusion is an old method for detecting 
microvessels. A large number of labs [8, 15, 17, 23, 27] still adopt it due to the following advantages: simplicity, affordability [5], applicability to a wide range of tissue, and suitability for quantification by stereological methods [30]. Unfortunately, ink dissolves quickly in aqueous solutions and easily flows away, which leads to the unsatisfactory filling of ink perfusion in large and small vessels. Additionally, ink perfusion is difficult to combine with other morphologic methods $[5,25,31]$. These disadvantages limited the use of simple ink perfusion. However, due to its above advantages, ink perfusion would be a powerful technique to study retinopathies if the disadvantages of the technique were overcome.

Gelatin is an extractive from the collegen inside animals' connective tissue, which can be dissolved in water at temperatures above $40^{\circ} \mathrm{C}$ and then coagulated into a gel at temperatures below $30^{\circ} \mathrm{C}$. Using this feature of gelatin, Tata et al. combined the ink perfusion with gelatin and perfused the ink-gelatin mixture into rat brain to investigate brain capillary structure [30]. However, the question is still open whether the gelatin-ink perfusion overcomes the above disadvantages of simple ink perfusion and can be used to fill completely the rat retinal microvessels, an end arterial system which is quite different from the network of the brain microvessels. In this study, we tried to optimize the gelatinink perfusion method of rat retinae and tested whether the labeling was comparable to the widely used von Willebrand factor (vWf) immunostaining for the rat retina. We also investigated whether this labeling technique affected the following labeling of retinal neurons and glial cells in the same tissue.

\section{Material and Methods}

\section{Animals}

Ninety adult Sprague-Dawley rats (200-250 g) were used in this study (purchased from Central South University, P.R.C.). All animals were housed under controlled environmental conditions on a $12 \mathrm{hr}$ light/dark cycle with ad libitum access to food and water. All protocols were approved by the local animal ethics committee and were in conformance with the Chinese government animal protection and management law and the guidelines on animal experiments at our university.

The design of the experiments. Firstly, 60 rats were subjected to 20 combinations ( $\mathrm{n}=3$ in each combination) of different gelatin concentrations $(0 \%, 1 \%, 3 \%$ and $5 \%)$ and perfusion pressures $(100 \%, 120 \%, 140 \%, 160 \%$ and $180 \%$ rat mean arterial pressure (MAP)) during retinal perfusion. Based on the data from these 60 rats, we found that perfusion with $3 \%$ gelatin-ink at a perfusion pressure of $140 \%$ rat MAP completely filled the peripheral retinal vessel lumen, but only partially filled the central vessels; in contrast, perfusion conditions of 5\% gelatin-ink at a perfusion pressure of $180 \%$ rat MAP more completely filled the central retinal vessel lumen, but not the peripheral vessels. Hence, we hypothesized that a combination of the two perfusion condi- tions (3\% and 5\% gelatin-ink mixtures) would completely fill the central and peripheral retinal vessel lumen and would be the optimal condition for the ink-gelatin infusion method. Secondly, 12 rats were used for evaluation of the retinal vessel labeling outcome of the gelatin-ink perfusion method under the optimal condition $(n=6)$ by using the $\mathrm{vWf}$ immunostaining $(n=6)$ as control. Finally, in order to investigate whether this improved labeling technique affected the following labeling of retinal neurons and glial cells in the same tissue, 18 rats were randomly divided into gelatinink $(n=6)$, control $(n=6)$ and fluorogold group $(n=6)$.

\section{Preparation of gelatin-ink mixture}

The Chinese ink and gelatin were purchased from the Yi De Ge Ink Company (Beijing, China) and the Shanghai Chemical Reagent Corp. (Shanghai, China), respectively. The gelatin was dissolved in double distilled water at $50^{\circ} \mathrm{C}$, being made up of different concentrations of the gelatin solutions. The pure ink and each concentration of gelatin solution were then mixed at $1: 1$ in volume to serve as the final perfusion fluid.

\section{Infusion of ink-gelatin mixture and retinal tissue preparation}

We used methods described previously [6], with slight modifications. The systolic blood pressure of each conscious rat was measured using the tailcuff method with an electrosphygmomanometer (BL-New Century, Chengdu, China). The MAP for each rat was averaged from three separate measurements. The MAP for all rats was $113 \pm 7 \mathrm{mmHg}$.

Under deep anesthesia, the thoracic cavity of the rat was opened, and the right atrium was cut to ensure outflow. Blood washout was achieved with a left ventricular puncture using a 12-gauge needle and a constant pressure perfusion assembly. The washout medium was $0.9 \%$ saline at $37^{\circ} \mathrm{C}$. The abdominal aorta was ligated just under the diaphragm. When the fluid flowing out of the right atrium was clear, rats were infused with a gelatin-ink mixture at a perfusion pressure based on the MAP at $37^{\circ} \mathrm{C}$. The superior vena was ligated after the ink was observed exiting from the right atrium, and the thoracic aorta was ligated at the end of the infusion. Successful perfusion was indicated by marked blackening of the eyes, snout and ears. In order to coagulate the gelatin-ink mixture, the rats were placed at $4{ }^{\circ} \mathrm{C}$ for 40 $\min$. Then the eyes were enucleated, the corneas were incised, and the lenses removed. The retina, or the remainder of the eye, was post-fixed in 4\% paraformaldehyde for $4 \mathrm{hr}$ at $4^{\circ} \mathrm{C}$ and then immersed in $30 \%$ sucrose. Cross sections through the optic disc of the left retinae were cut at a thickness of $14 \mu \mathrm{m}$ by a cryostat machine, mounted on glass slides, dried at room temperature, and stored at $-20^{\circ} \mathrm{C}$. Whole-mounts of right retinae were prepared on a glass slides, dried at room temperature, and stored at $-20^{\circ} \mathrm{C}$.

\section{Criteria to evaluate filling effect of ink-gelatin perfusion rat retinal microvessels}

The filling effect of ink-gelatin perfusion includes: (i) continuity of vascular trunks and branches; (ii) the homo- 
geneity of ink-gelatin filled cavity of different-size vessels; (iii) a cut-off border of the different-size vessels.

\section{Tissue preparation of retina without the gelatin-ink perfusion}

Under deep anesthesia, rats were perfused at rat MAP through the left ventricle with $0.9 \%$ saline followed by 500 $\mathrm{ml} \mathrm{4 \%}$ paraformaldehyde in $0.1 \mathrm{M}$ phosphate buffer (PB, $\mathrm{pH}$ 7.4). Both right and left eyes were enucleated. Other methods were the same as those described above.

\section{Retinal ganglion cell labeling with fluorogold}

Slight modifications were made to this previously described technique [26]. The rat was anesthetized peritoneally with $10 \%$ chloral hydrate $(0.4 \mu \mathrm{l} / \mathrm{kg})$. Both superior colliculi were exposed, and the pia overlying the superior colliculus was removed. A piece of gelfoam soaked in a solution of $5 \%$ fluorogold (Fluorochrome, LLC) and 10\% dimethylsulphoxide in saline was placed on top of the superior colliculus, and the skin was sutured. Seven days later, the animals were first infused with $20 \mathrm{ml}$ of $37^{\circ} \mathrm{C}$ ink containing $3 \%$ gelatin at $140 \%$ rat MAP, and then with $20 \mathrm{ml}$ of $37^{\circ} \mathrm{C}$ ink containing $5 \%$ gelatin at $180 \%$ rat MAP. Whole mount retinae were observed using fluorescence microscopy. Peripheral and central retinal cells, which were labeled with fluorogold were photographed.

\section{Immunofluorescence}

For immunofluorescence experiments, sections and whole retinae that were stored at $-20^{\circ} \mathrm{C}$ were warmed to room temperature and rehydrated with $0.01 \mathrm{M}$ phosphate buffered saline (PBS) for $10 \mathrm{~min}$. The samples were incubated in blocking solution $(5 \%$ BSA and $0.3 \%$ Triton $\mathrm{X}$ 100 in $0.1 \mathrm{M} \mathrm{PB}$ ) for $2 \mathrm{hr}$ at room temperature. Then the sections were incubated in rabbit antiserum directed against von Willebrand factor (vWf) (1:200, Chemicon) overnight at $4^{\circ} \mathrm{C}$. The whole retinae were incubated in the same antisera to $\mathrm{vWf}(1: 500)$ for $48 \mathrm{hr}$ at $4^{\circ} \mathrm{C}$. After three washes in PBS, the sections were incubated in the corresponding secondary antibodies (1:200, Vector) for $1.5 \mathrm{hr}$ at room temperature. The whole retinae were incubated in the same secondary antibodies $\left(1: 200\right.$, Vector) overnight at $4^{\circ} \mathrm{C}$. Following a PBS wash, the sections were incubated in fluorescein isothiocyanate (FITC)-conjugated-anti-rabbit $\operatorname{IgG} \quad(1: 200$, Vector) for $1 \mathrm{hr}$ at room temperature. The whole retinae were incubated in the same $\operatorname{IgG}(1: 200)$ for $3 \mathrm{hr}$ at room temperature. After three washes, the sections and whole retinae were dried at room temperature for $5 \mathrm{~min}$ and coverslipped with an aqueous mount. As negative controls, an adjacent series of sections were processed using the same procedures without the primary antibodies.

\section{Immunohistochemistry}

Sections stored at $-20^{\circ} \mathrm{C}$ were warmed up to room temperature, rehydrated and incubated in $0.3 \%$ hydrogen peroxide for $15 \mathrm{~min}$ to block endogenous peroxidase activity. After incubation in blocking solution (5\% BSA and $0.3 \%$ Triton X-100 in P.B.) for $1 \mathrm{hr}$ at room temperature, the sections were incubated in polyclonal anti-rat antibodies against either NeuN (raised in mouse, 1:200, Chemicon), parvalbumin (raised in mouse, 1:2000, Chemicon) or glial fibrillary acidic protein (GFAP, raised in rabbit, 1:1000, Vector) overnight at $4{ }^{\circ} \mathrm{C}$. After three washes with PBS, the sections were incubated in the corresponding secondary antibodies (1:200, Vector) for $1.5 \mathrm{hr}$ at room temperature. Then, the sections were incubated in the avidin-biotinperoxidase complex (ABC) reagent (1:200, Vector) for $1 \mathrm{hr}$ at room temperature, rinsed and stained with $0.05 \% 3,3^{\prime}-$ diaminobenzidine (DAB, Sigma) in the presence of $0.01 \%$ hydrogen peroxide. As negative controls, an adjacent series of sections were processed using the same procedures by removing primary antibodies. All sections were dried, dehydrated and coverslipped.

\section{Results}

\section{Optimization of the gelatin-ink infusion technique}

In previous reports, the effects of ink perfusion varied greatly with perfusion methods $[1,5,19,29,31]$. In order to optimize the gelatin-ink infusion of rat retinae, we chose an ink temperature of $37^{\circ} \mathrm{C}$, based on the physico-chemical properties of gelatin and rat body temperature, and four concentration gelatin-ink $(0 \%, 1 \%, 3 \%$ and $5 \%$ gelatin in ink) with five perfusion pressures $(100 \%, 120 \%, 140 \%, 160 \%$ and $180 \%$ rat MAP).

Ink alone or $1 \%$ gelatin-ink perfusion at $100 \%, 120 \%$, $140 \%, 160 \%$ or $180 \%$ rat MAP showed the discontinuity of central and peripheral vessels and a poor filling in the central and peripheral retinal vessel lumen (data not shown). Three
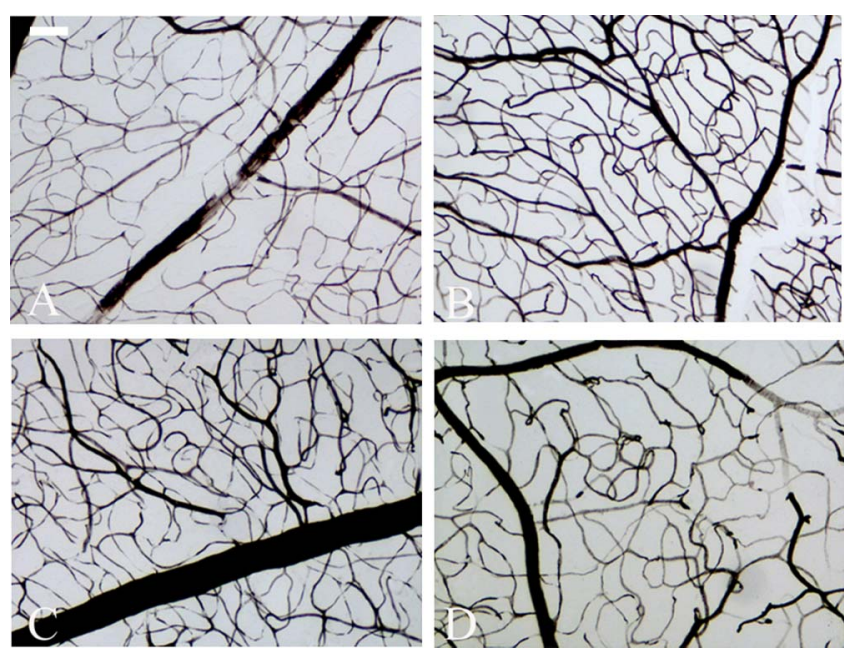

Fig. 1. Retinal blood vessel labeling with $3 \%$ gelatin-ink or $5 \%$ gelatin-ink infusion. In the whole-mounted rat retinal specimens that were infused with the $3 \%$ gelatin-ink mixture at a perfusion pressure of $140 \%$ rat MAP, the peripheral retinal vessels (B) were completely filled, but only partial central vessels (A). In contrast, perfusion conditions of $5 \%$ gelatin-ink at a perfusion pressure of $180 \%$ rat MAP filled the central retinal vessels $($ C), but not the peripheral vessels $(\mathbf{D})$. Bar $=100 \mu \mathrm{m}$. 
percent gelatin-ink infusion did achieve a complete filling of peripheral retinal vessel lumen with a satisfactory vascular continuity when the perfusion pressure reached $140 \%$ rat MAP (Fig. 1B), but the filling of the central vessels, just like that of Figure 1A, was unsatisfactory. Similar results were observed under conditions of 3\% gelatin-ink combined with $160 \%$ or $180 \%$ rat MAP. In contrast, perfusion conditions of $5 \%$ gelatin-ink at a perfusion pressure of $180 \%$ rat MAP more completely filled the central retinal vessel lumen, but not the peripheral vessels (Fig. 1C and D). Under conditions using 5\% gelatin-ink at the rest pressures, due to the high viscosity of $5 \%$ gelatin-ink, a less filling effect was achieved in central and peripheral vessels when compared to that in the condition using 5\% gelatin-ink at $180 \%$ rat MAP (data not shown).

Based on the above results, we hypothesized that a combination of the two perfusion conditions (3\% and $5 \%$ gelatin-ink mixtures) would completely fill the central and peripheral retinal vessel lumen. To test this hypothesis, rats were first infused with $20 \mathrm{ml}$ of $37^{\circ} \mathrm{C}$ ink plus $3 \%$ gelatin at $140 \%$ rat MAP. Then the rats were infused with $20 \mathrm{ml}$ of $37^{\circ} \mathrm{C}$ ink plus $5 \%$ gelatin at $180 \%$ rat MAP. As shown in Figures $2 \mathrm{~A}$ and $\mathrm{B}$, both the central and peripheral vessels showed a satisfactory continuity and the lumen were completely filled. Moreover, there existed no ink leakage into the retina and thus the contrast between the vessels and surrounding tissue was improved dramatically.

\section{Comparison of vessel labeling with gelatin-ink infusion or vWf immunostaining}

In whole-mount retinae, the peripheral vessels stained by vWf were clear networks (Fig. 2E), with only a few small branches of vessels in the central part of the retina (Fig. 2D). Background staining was substantial. In the retinal sections, only large vessels were labeled in the nerve fiber layer, inner nuclear layer and outer plexiform layer (Fig. 2F).

In contrast, in the whole-mount samples treated with gelatin-ink infusions, the central and peripheral vessels appeared as clear, homogeneous, black networks (Fig. 2A and B). The vessels were distinct in contrast with the surrounding tissues. In the retinal sections, black cross-sections of vessels with distinct borders were observed in the nerve fiber layer, inner nuclear layer and outer plexiform layer (Fig. 2C). The number of gelatin-ink labeled vessels was significantly larger than that marked by $\mathrm{vWf}$ (Fig. 2F).

\section{Double labeling with gelatin-ink infusion and immunohistochemistry}

$\mathrm{NeuN}$ and parvalbumin are markers for neurons, and GFAP is a marker for astrocytes. Our immunostaining results for rat retina were similar to those of other studies [7, $12,32]$. NeuN-labeled neurons were observed mainly in the ganglion cell layer (Fig. 3A). Parvalbumin immunoreactivity was observed mainly in the retinal inner part, and in particular in the inner nuclear layer (Fig. 3B). In contrast,
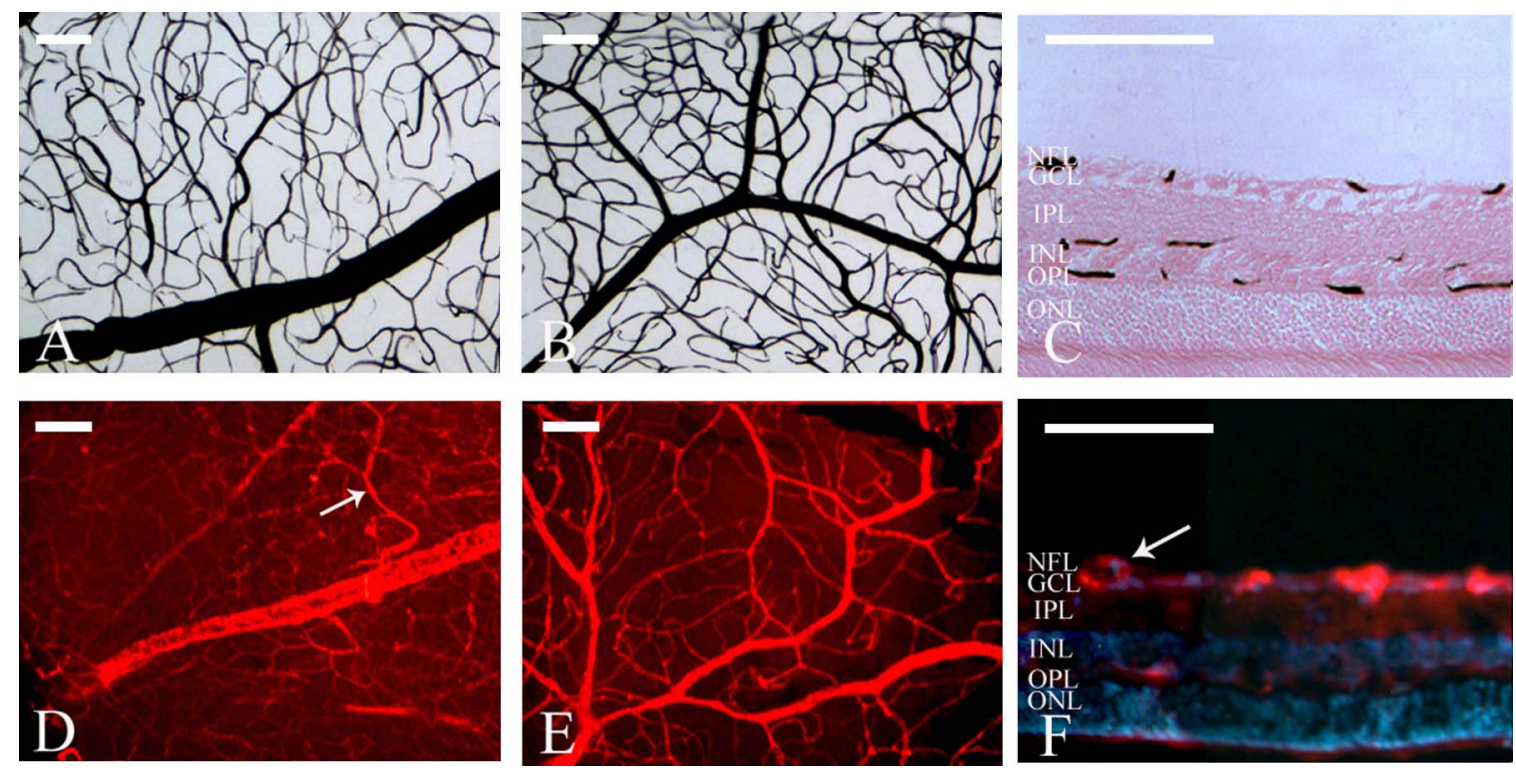

Fig. 2. Retinal blood vessels labeling with gelatin-ink infusion or with vWf immunostaining. In the whole-mounted rat retinal specimens that were infused with the gelatin-ink mixture, the central (A) and peripheral (B) vessels were visible as distinct, homogenous, black networks. (C) In rat retinal sections infused with gelatin-ink, black vessels with clear borders were mainly observed in the inner retina. Peripheral vessels (E) labeled by vWf were visible in the whole-mounted retina, while only a few branches were labeled in central vessels (D). The arrow (D) showed the clearly stained small branch of vessels. The number of vessels labeled by vWf $(\mathbf{F})$ in the rat retinal section was obviously lower than that labeled by gelatin-ink infusion $(\mathbf{C})$. The arrow $(\mathbf{F})$ showed the large vessel. Red, vWf immunofluorescence; Blue, Hoechst nuclear staining; NFL, nerve fiber layer; GCL, ganglion cell layer; IPL, inner plexiform layer; INL, inner nuclear layer; OPL, outer plexiform layer; ONL, outer nuclear layer. Bar $=100 \mu \mathrm{m}$. 

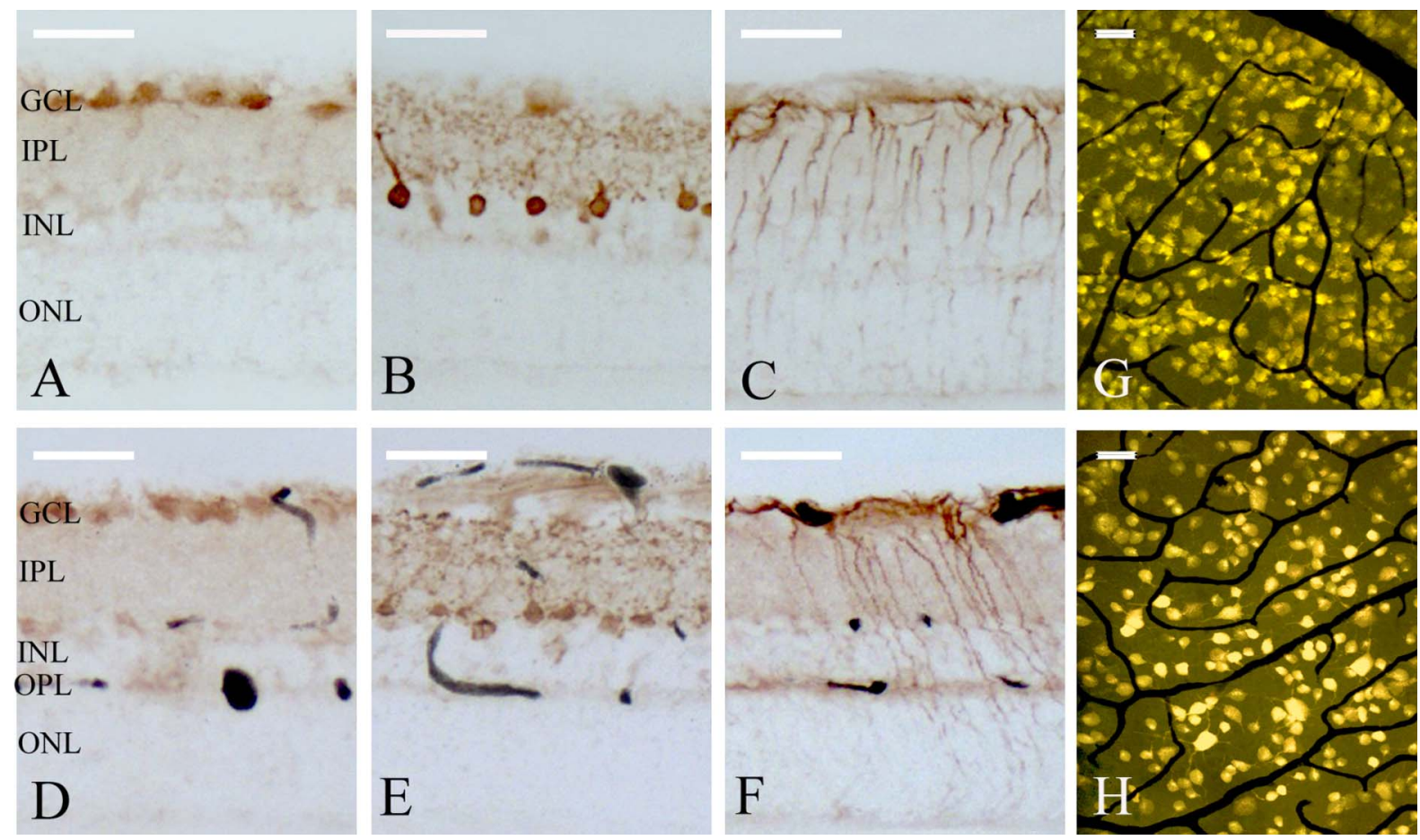

Fig. 3. Double labeling with gelatin-ink infusion and either immunohistochemistry or fluorogold labeling. In the rat retina, NeuN-labeled neurons (A) were observed mainly in the ganglion cell layer, parvalbumin immunoreactivity (B) was observed in the retinal inner layer, and GFAP immunoreactivity $(\mathbf{C})$ was present in glial cell processes. Retinae infused with gelatin-ink resulted in filled vessels, which appear as black cross-sections (D-F). The immunoreactivity of NeuN (D), parvalbumin (E) and GFAP (F) were similar to those of retinae without gelatin-ink infusion. Double labeling of gelatin-ink infusion with fluorogold in the central (G) and peripheral (H) in the whole mount of rat retinae show good contrast between vessels (black) and retinal ganglion cells (yellow). Black: vessel sections perfused with gelatin-ink. GCL, ganglion cell layer; IPL, inner plexiform layer; INL, inner nuclear layer; OPL, outer plexiform layer; ONL, outer nuclear layer. Bar=40 $\mu$ m.

GFAP immunoreactivity was mainly located in glial cell processes, which extended from the ganglion cell layer to the outer plexiform layer and even to the outer nuclear layer (Fig. 3C).

In the retina treated with gelatin-ink, many black crosssections of vessels were filled. The immunohistochemistry results for NeuN (Fig. 3D), parvalbumin (Fig. 3E) and GFAP (Fig. 3F) in samples treated with gelatin-ink infusion were similar to results in untreated retinae. In these experiments, gelatin-ink infusion had no effect on the immunostaining of retinal neurons and glial cells.

\section{Double labeling with gelatin-ink infusion and fluorogold}

Fluorogold retrograde labeling is a commonly used marker for retinal ganglion cells. After gelatin-ink perfusion, fluorogold labeled retinal ganglion cells were well-visualized both in the central (Fig. 3G) and peripheral (Fig. 3H) part of retinae. The double labeling provided a strategy to precisely visualize the spatial relationship between retinal ganglion cells and vessels.

\section{Discussion}

The aim of this study was to improve the gelatin-ink perfusion method to better visualize rat retinal microvessels and extend the practical scale of this classic method. In order to accomplish this aim, we tested twenty combinations by using four kinds of gelatin concentration and five kinds of perfusion pressure. We found that 3\% gelatin-ink with the perfusion pressure of $140 \%$ rat MAP and then $5 \%$ gelatinink mixtures with the perfusion pressure of $180 \%$ rat MAP, perfectly labeled both central and peripheral vessels based on the evaluation standard. Then, we used vWf immunostaining to further evaluate the labeling effect of the newlyimproved method.

vWf is widely used as a marker of vessels in all kinds of archival tissues, however expression of vWf depends on the fuctional status of the endothelium [20] and also varies with different sized vessels. For example, Barou seldom detected vWF positive staining in the capillaries in bone [1]. Similarly, in our studies of retinal sections, vWf immunostaining mainly appeared in the larger vessels of the nerve fiber layer, inner nuclear layer and outer plexiform layer, and sparsely in capillaries. The different staining characteristics of larger vessels and capillaries in our study may reflect differences in vWf expression levels. Additionally, we also found that small peripheral and central vessels were not clearly marked by vWfat the same time. This vWflabeling pattern was similar to that of laminin, another vessel marker, and pepsin digestion was shown to increase laminin labeling [2]. Hence, the 
uneven vWf labeling observed in peripheral and central vessels may result from the fact that the thickness of the nerve fiber layer is thick in the central part and comparatively thin in the peripheral part of the retina. This unique anatomic feature led to a poor penetration of anti-vWf antibody in central vessels and a better penetration in peripheral vessels during the immunostaining. Although application of tissue-digestive regeants (like pepsin) will improve the permeability of the central retina, it will also non-selectively destroy the comparatively appropriate permeability of the peripheral retina. We concluded that standard vWf immunostaining cannot efficiently label all retinal vessels.

In the present study, the central and peripheral retinal vessels of all size ranges were labeled with gelatin-ink perfusion in both whole-mounts and sections. No ink leakage into the retina occurred, and the contrast between the vessels and the surrounding tissues was dramatically improved. Gelatin-ink perfusion may be used for complete visualization of rat retinal microvessels, overcoming the disadvantages of vWf immunostaining and only ink perfusion. Researchers can use this technique to accurately monitor and measure changes in vascular parameters, such as microvessel area and density [29, 30].

There is a close relationship between neural activity and vascular perfusion in the retina. Thus, assessing both vascular and neural parameters in the same retinal section or whole mount is very advantageous. Our results showed that gelatin-ink infusion could double label with either immunostaining or fluorogold labeling with a striking contrast. This allows visualization of the close spatial relationship between vessels and neurons and glial cells. Additionally, this double labeling technique could be used to precisely measure microvessel area [1, 22], microvessel density [5], neuron number [30], glial cell number or glial cell area [13] in the same retina. These measurements would allow for simple, cost-efficient investigations of the relationship between microvascular changes and neuron and glial cell changes in retinopathies. Regretfully, the gelatin-ink infusion method requires a perfusion procedure before tissue sampling. Therefore the application of this method is limited to the animal experimental system, but not to the tissue sections obtained from human pathological specimens.

In conclusion, gelatin-ink infusion is a useful method for morphological characterization of rat retinal microvessels. Gelatin-ink infusion can be coupled with fluorogold or immunostaining to successfully double label tissue. This technique has wide applicability for labeling the retinal vasculature in both healthy and pathological states.

\section{Acknowledgements}

This work was supported by grants from the National Natural Science Foundation of China (No. 30100098 and No. 30570979).

\section{References}

1. Barou, O., Mekraldi, S., Vico, L., Boivin, G., Alexandre, C. and Lafage-Proust, M. H. (2002) Relationships between trabecular bone remodeling and bone vascularization: a quantitative study. Bone 30; 604-612.

2. Belford, D. A., Gole, G. A. and Rush, R. A. (1987) Localization of laminin to retinal vessels of the rat and mouse using whole mounts. Invest. Ophthalmol. Vis. Sci. 28; 1761-1766.

3. Bell, M. A. and Scarrow, W. G. (1984) Staining for microvascular alkaline phosphatase in thick celloidin sections of nervous tissue: morphometric and pathological applications. Microvasc. Res. 27; 189-203.

4. Ben-Nun, J., Alder, V. A., Constable, I. J. and Roberts, C. E. (1990) The patency of the retinal vasculature to erythrocytes in retinal vascular disease. Invest. Ophthalmol. Vis. Sci. 31; 464470 .

5. Browning, J., Wylie, C. K. and Gole, G. (1997) Quantification of oxygen-induced retinopathy in the mouse. Invest. Ophthalmol. Vis. Sci. 38; 1168-1174.

6. Deng, P. Y., Ye, F., Zhu, H. Q., Cai, W. J., Deng, H. W. and Li, Y. J. (2003) An increase in the synthesis and release of calcitonin gene-related peptide in two-kidney, one-clip hypertensive rats. Regul. Pept. 114; 175-182.

7. Dijk, F., Bergen, A. and Kamphuis, W. (2007) GAP-43 expression is upregulated in retinal ganglion cells after ischemia/reperfusion-induced damage. Exp. Eye Res. 84; 858-867.

8. Finger, S. and Dunnett, B. S. (1989) Nimodipine enhances growth and vascularization of neural grafts. Exp. Neurol. 104; 19.

9. Gariano, R. F., Iruela-Arispe, M. L., Sage, E. H. and Hendrickson, A. E. (1996) Immunohistochemical characterization of developing and mature primate retinal blood vessels. Invest. Ophthalmol. Vis. Sci. 37; 93-103.

10. Hudetz, A. G. (1999) Mathematical model of oxygen transport in the cerebral cortex. Brain Res. 817; 75-83.

11. Hudetz, A. G., Feher, G., Weigle, C. G., Knuese, D. E. and Kampine, J. P. (1995) Video microscopy of cerebrocortical capillary flow: response to hypotension and intracranial hypertension. Am. J. Physiol. 268; H2202-2210.

12. Jin, Y., Zhong, Y. M. and Yang, X. L. (2007) Natriuretic peptides are localized to rat retinal amacrine cells. Neurosci. Lett. 421; 106-109.

13. Kalinin, S., Gavrilyuk, V., Polak, P. E., Vasser, R., Zhao, J., Heneka, M. T. and Feinstein, D. L. (2007) Noradrenaline deficiency in brain increases beta-amyloid plaque burden in an animal model of Alzheimer's disease. Neurobiol. Aging 28; 1206-1214.

14. Klima, K. A. (2006) Focus on fluorescein angiography. Insight $31 ; 15-17$.

15. Korol, D. L. and Brunjes, P. C. (1992) Unilateral naris closure and vascular development in the rat olfactory bulb. Neuroscience 46; 631-641.

16. Larrazabal, L. I. and Penn, J. S. (1990) Fluorescein angiography of the newborn rat. Implications in oxygen-induced retinopathy. Invest. Ophthalmol. Vis. Sci. 31; 810-818.

17. Lawrence, J. M., Huang, S. K. and Raisman, G. (1984) Vascular and astrocytic reactions during establishment of hippocampal transplants in adult host brain. Neuroscience 12; 745-760.

18. Lee, G. D., Aruna, J. H., Barrett, P. M., Lei, D. L., Ingram, D. K. and Mouton, P. R. (2005) Stereological analysis of microvascular parameters in a double transgenic model of Alzheimer's disease. Brain Res. Bull. 65; 317-322.

19. Li, L., Sun, J., Li, M. and Ye, Z. (2004) The perfusion method for making brain vessel model by Chinese ink combined with gelatin on the warm condition. Chin. J. Clin. Anat. 22; 224.

20. Lip, G. Y. and Blann, A. (1997) Von Willebrand factor: a marker of endothelial dysfunction in vascular disorders? Cardiovasc. 
Res. 34; 255-265.

21. Liu, Y., Liang, X., Xu, C., Xie, S., Kuang, W. and Liu, Z. (2006) Quantification of oxygen-induced retinopathy in the mouse. Yan Ke Xue Bao (Chinese Journal) 22; 103-106, 124.

22. Lofqvist, C., Chen, J., Connor, K. M., Smith, A. C., Aderman, C. M., Liu, N., Pintar, J. E., Ludwig, T., Hellstrom, A. and Smith, L. E. (2007) IGFBP3 suppresses retinopathy through suppression of oxygen-induced vessel loss and promotion of vascular regrowth. Proc. Natl. Acad. Sci. U S A 104; 10589-10594.

23. Miyoshi, Y., Date, I. and Ohmoto, T. (1995) Three-dimensional morphological study of microvascular regeneration in cavity wall of the rat cerebral cortex using the scanning electron microscope: implications for delayed neural grafting into brain cavities. Exp. Neurol. 131; 69-82.

24. Pawlik, G., Rackl, A. and Bing, R. (1981) The quantitative capillary topography and blood flow in the cerebral cortex of cats: an in vivo microscopic study. Brain Res. 208; 35-58.

25. Pen, X. Y., Chen, D. Y., Yan, M. and Den, J. (2000) A quantifying model of vascular proliferation in oxygen-induced retinopathy. Chin. J. Ocul. Fundus Dis. 16; 260-263.

26. Piri, N., Song, M., Kwong, J. M. K. and Caprioli, J. (2007) Modulation of alpha and beta crystallin expression in rat retinas with ocular hypertension-induced ganglion cell degeneration. Brain Res. 1141; 1-9.

27. Sirevaag, A. M., Black, J. E., Shafron, D. and Greenough, W. T.
(1988) Direct evidence that complex experience increases capillary branching and surface area in visual cortex of young rats. Brain Res. 471; 299-304.

28. Strauss, R. W., Rombold, F., Kampik, A. and Neubauer, A. S. (2007) Fluorescein angiography compared to three-dimensional measurements by the retinal thickness analyzer in classic choroidal neovascularization. Ophthalmic Res. 39; 98-102.

29. Taguchi, Y., Takashima, S., Sasahara, E., Inoue, H. and Ohtani, O. (2004) Morphological changes in capillaries in the ischemic brain in Wistar rats. Arch. Histol. Cytol. 67; 253-261.

30. Tata, D. A. and Anderson, B. J. (2002) A new method for the investigation of capillary structure. J. Neurosci. Methods 113; 199206.

31. Xu, H. B. and Chen, X. L. (2003) Effect of oxygen inhalation on the retina of newborn rats and its mechanism. Chin. J. Ocul. Fundus Dis. 19; 306-309.

32. Xue, L. P., Lu, J., Cao, Q., Hu, S., Ding, P. and Ling, E. A. (2006) Müller glial cells express nestin coupled with glial fibrillary acidic protein in experimentally induced glaucoma in the rat retina. Neuroscience $139 ; 723-732$.

This is an open access article distributed under the Creative Commons Attribution License, which permits unrestricted use, distribution, and reproduction in any medium, provided the original work is properly cited. 